\title{
Glucosamine increases the expression of YKL-40 and osteogenic marker genes in hMSC during osteogenic differentiation
}

\author{
Ramona Lieder, ${ }^{\mathrm{a}, \mathrm{b}}$ Sigrídur Thóra ReynisdótTIR, ${ }^{\mathrm{a}}$ Finnbogi Thormódsson, ${ }^{\mathrm{e}}$ Chuen-How NG,${ }^{\mathrm{c}}$ Jon \\ Magnús EINARSSON, ${ }^{\mathrm{c}}$ Jóhannes Gíslason, ${ }^{\mathrm{c}}$ Jóhannes BJÖRnSSON, ${ }^{\mathrm{d}, \mathrm{f}}$ Sveinn GudMUNDSSON, ${ }^{\mathrm{a}}$ Pétur Henry \\ PETERSEN, ${ }^{\mathrm{e}}$ and Ólafur Eysteinn SIGURJÓNSSON ${ }^{\mathrm{a}, \mathrm{b}, \mathrm{d}, *}$
}

\author{
${ }^{a}$ The Blood Bank, Landspitali University Hospital, Snorrabraut 60, 105 Reykjavik, Iceland \\ ${ }^{\mathrm{b}}$ School of Science and Engineering, Reykjavik University, Menntavegi 1, 101 Reykjavik, Iceland \\ ${ }^{\mathrm{c}}$ Genis ehf, Vatnagördum 18, 104 Reykjavik, Iceland \\ ${ }^{\mathrm{d}}$ Biomedical Center, University of Iceland, Vatnsmyrarvegi 16, 101 Reykjavik, Iceland \\ e Department of Anatomy, Medical Faculty, University of Iceland, Vatnsmyrarvegi 16, 101 Reykjavik, Iceland \\ ${ }^{\mathrm{f}}$ Department of Pathology, Landspitali University Hospital, Baronsstig, 101 Reykjavik, Iceland
}

Received 28 February 2012; Accepted 25 March 2012

(C) The Author(s) 2012. This article is published with open access at Springerlink.com

\begin{abstract}
Human mesenchymal stem cells (hMSC) can be expanded in vitro and differentiated towards osteogenic, chondrogenic or adipogenic lineages, making them an attractive source for tissue engineering and regenerative medicine. Chitinase-like-proteins (CLPs) belong to the family 18 glycosyl hydrolases and are believed to play a role in inflammation and tissue remodelling. The aim of this study was to determine the effect of the aminosugar glucosamine on the expression of the CLP YKL- 40 during osteogenic differentiation of hMSC. Glucosamine did not affect multipotency of hMSC nor proliferation rate of undifferentiated hMSC. YKL-40 was expressed during both expansion of undifferentiated hMSC and during osteogenic differentiation. A slight but nonsignificant increase in YKL-40 expression was observed with glucosamine, accompanied by a pH-dependent delay in mineralization. However, glucosamine induced higher expression of osteogenic marker genes.
\end{abstract}

Keywords: YKL-40, mesenchymal stem cells, osteogenic differentiation, chitinase-like-protein

\section{Introduction}

Stem cells hold a great promise as a tool in basic research and development of drugs and new treatments in regenerative medicine. Human multipotent mesenchymal stromal cells (hMSC) is a commonly used term for a heterogeneous population of cells that can be derived from human bone marrow, adipose tissue, peripheral blood or umbilical cord blood $^{1,2}$. These cells can differentiate into cells of the mesenchymal lineage, e.g. to osteoblasts, chondrocytes, fibroblasts and adipocytes, as well as, stromal cells of the bone marrow ${ }^{3}$.

In connection to the regeneration of the osteogenic matrix, chitinases and chitinase-like-proteins (CLP) have been suggested to have a role in defence mechanisms against chitincontaining particles or organisms and to participate in tissue remodelling and inflammation ${ }^{4,5}$. Whether triggering of the immune response and the potential role in tissue remodelling is directly affected by the chitinase-like-proteins or dependent on

*To whom correspondence should be addressed. E-mail: oes@landspitali.is the downstream induction of cytokines and growth factors has not been investigated ${ }^{5}$. There have been indications that the chitinase-like-proteins are expressed during tissue damage or regeneration in an attempt to counter the negative effect of inflammatory cytokines ${ }^{6}$. In humans, three chitinase-likeproteins are known, YKL-39, YKL-40 and oviductin, as well as the active chitinases acidic mammalian chitinase (AMCase) and chitotriosidase ${ }^{7}$. The active chitinases belong to the family 18 glycosyl hydrolases, the same family as the CLPs. CLPs have lost the ability to degrade chitin due to an amino acid substitution in the binding cleft ${ }^{7,8}$, but the ability to bind chitin and chitooligosaccharides is still retained ${ }^{9}$. Binding of the ligand induces a large conformational change in YKL-40 which indicates a potential signalling role of the enzyme ${ }^{10}$. YKL-40 is expressed by articular chondrocytes, synoviocytes, osteoblasts and differentiated macrophages and is up-regulated in inflammatory diseases like rheumatoid arthritis and osteoarthritis $^{6,11}$. YKL-40 expression has been suggested to be regulated by the transcription factor NF- $\kappa B^{-1}{ }^{6}$. Also, it has been proposed that YKL-40 might prevent damage to the extra-cellular matrix during inflammation by reducing the deleterious effect of pro-inflammatory cytokines ${ }^{12}$.

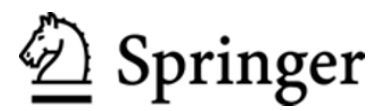


Due to chitinases, chitin, the second most abundant compound in nature, does not accumulate in the environment ${ }^{13}$. Chitin, a structural component in the cell wall of crustaceans and fungi, can be used for the production of glucosamine, chitooligosaccharides and chitosan, which have wide ranging uses in biotechology, agriculture and food science ${ }^{13}$. Glucosamine is an aminosugar that naturally occurs in the body and has been claimed to have beneficial effects on osteoarthritis as it constitutes one of the subunits in glycosaminoglycans found in the extra-cellular matrix of cartilage $^{14}$. It is unknown how glucosamine might work to ameliorate arthritis, but a connection to nitric oxide production has been suggested ${ }^{14,15}$. The effect of glucosamine on bone resorption and remodelling has been evaluated in a collagenase-induced osteoarthritis model in mice ${ }^{16}$. It was shown that excessive bone formation and bone remodelling were reduced and bone resorption was decreased ${ }^{16}$. The effect of glucosamine on the CLPs and its role during osteogenesis of hMSC has not been investigated.

In this study, we show that the chitinase-like-protein YKL-40 is expressed in hMSC and is maintained during osteogenic differentiation of these cells. The effect of glucosamine on the expression of YKL-40 and osteogenic differentiation was analysed.

\section{Results and Discussion}

hMSC Morphology and Proliferation. Short-term expansion of cells (single passage) in control media and glucosamine supplemented media did not lead to changes in the expression of cell surface markers and the cells comply with the characteristics of bone-marrow derived mesenchymal stromal cells (supplementary Figure 1$)^{17}$. Cell morphology was typical of hMSC-small spindle-shaped cells attached to the surface of tissue culture plastic (Fig. 1A). Glucosamine did not affect cell attachment or morphology during expansion. During osteogenic differentiation, cell morphology changed to a more cuboidal and flattened shape. In later stages of differentiation, osteoblastic cells deposited calcium hydroxyapatite crystals and cell layers pulled together. No difference in phenotype was observed in the presence of glucosamine, except for the lack of calcium deposition.

Proliferation was similar during short-term expansion of hMSC in control media and glucosamine media, with a decrease in proliferation on days 5 and 7 (Fig. 1B).

Furthermore, the cells could be successfully differentiated towards the osteogenic, chondrogenic and adipogenic lineage, proving multi-lineage potential (supplementary Fig. 2).

Expression of the Chitinase-Like-Proteins (CLPs) in hMSC. CLPs have putative roles in defence mechanisms against chitin-containing particles and participate in tissue remodelling and inflammation ${ }^{4,5}$. Crystallography studies revealed that the enzyme can bind chitin and chitooligosaccharides with high affinity ${ }^{9,10}$. We therefore examined, whether the aminosugar glucosamine could affect the expression of the CLPs during short-term expansion of hMSC.

hMSC expressed the chitinase-like protein YKL-40 at day 3 and day 7 during expansion (supplementary Fig. 3). No statistical difference was found between control samples $(0.85$ \pm 0.85 fold increase over 7 days) and glucosamine treated

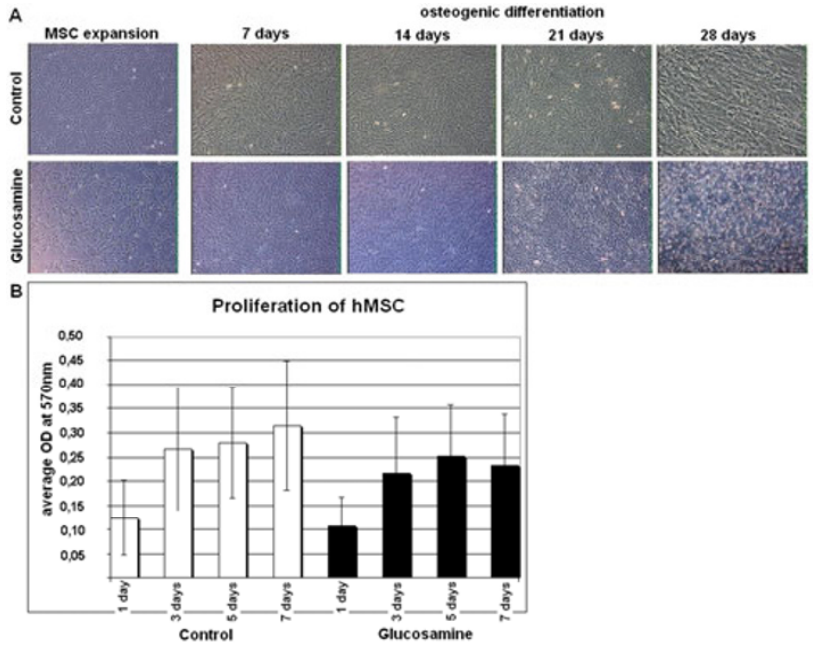

Figure 1. Effect of glucosamine on hMSC phenotype and proliferation. (A) Phenotype consistency in the expansion and osteogenic differentiation of hMSC (representative pictures); (B) Proliferation of hMSC grown in control media and media supplemented with $200 \mu \mathrm{g} / \mathrm{mL}$ Glucosamine. Bars illustrate proliferation on days $1,3,5$ and $7(n=42$ measurements).

samples (3.24 \pm 2.17 fold increase over 7 days) in the expression of YKL-40. The active chitinases, chitotriosidase (CHIT1) and acidic mammalian chitinase (AMCase) were not detected (data not shown).

During osteogenic differentiation, YKL-40 was expressed both in control $(1.82 \pm 0.53$ fold increase over 28 days $)$ and glucosamine treated samples $(3.52 \pm 0.63 ; \mathrm{p}=0.0828$ fold increase over 28 days) (Fig. 2A). A trend in which glucosamine increased YKL-40 expression during osteogenic differentiation was observed, but statistical significance $(\mathrm{p}<0.05)$ was not met.

Osteoblast Gene Expression. The following osteogenic marker genes: alkaline phosphatase (ALP), osteocalcin (OCN), osteopontin (OPN) and runt-related transcription factor 2 (RUNX-2) were expressed both in control and glucosamine treated samples during osteogenic differentiation (Fig. 2A). A statistically significant increase in the presence of glucosamine was observed for ALP $(0.63 \pm 0.24$ fold increase in control vs. $1.84 \pm 0.13$ fold increase in glucosamine; $p=0.0011)$, OCN $(1.41 \pm 0.41$ fold increase in control vs. $2.85 \pm 0.18$ fold increase in glucosamine; $p=0.0087)$ and RUNX-2 (1.06 \pm 0.31 fold increase in control vs. $2.39 \pm 0.17$ fold increase in glucosamine; $p=0.0037)$ gene expression. The difference in OPN expression $(0.20 \pm 0.41$ fold increase in control vs. 1.56 \pm 0.62 fold increase in glucosamine; $p=0.097$ ) was not statistically significant but a trend was observed that suggested glucosamine could increase the expression of OPN.

To visualize the correlation between the expression of YKL-40 and the expression of the osteogenic marker genes, a heatmap was generated (Fig. 2B) ${ }^{18}$. Clustering divided the sample population into three distinct groups: (1) control samples from day 7 to day 25 of osteogenic differentiation; (2) glucosamine treated samples from day 7 to day 21; and (3) late osteogenesis including control samples at day 28 and glucosamine treated samples from day 25 to day 28 . In the first

\section{是 Springer}


A

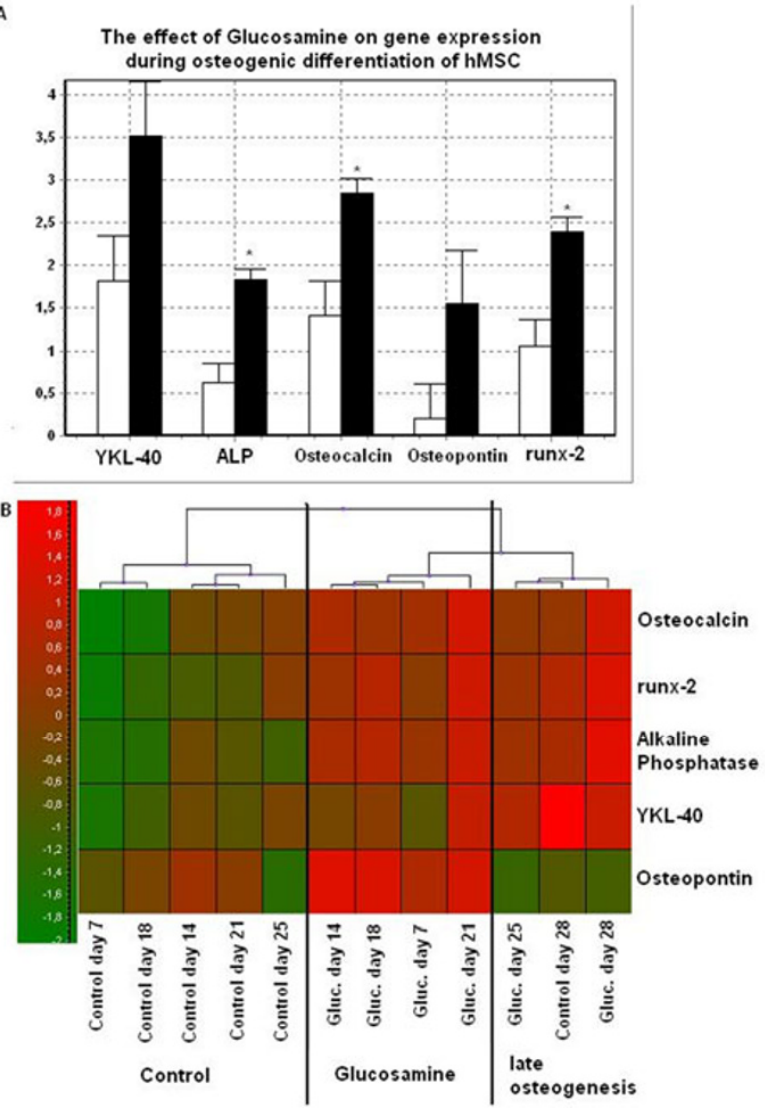

Figure 2. Effect of glucosamine on gene expression during osteogenic differentiation of hMSC. (A) Average expression of YKL-40, Alkaline Phosphatase (ALP), Osteocalcin (OCN), Osteopontin (OPN) and runt-related transcription factor 2 (RUNX-2) in control (white bars) and glucosamine (black bars) supplemented cultures over a period of 28 days of osteogenic differentiation. Bars describe average fold change with standard error. $\mathrm{n}=2 ; *=\mathrm{p}<0.05$; (B) Heatmap and hierarchical clustering of gene expression data auto-scaled to genes. Red colour depicts high expression and green colour depicts low expression $(n=2)$.

group overall expression of genes was low, except for the expression of OPN. The second group, i.e. early glucosamine treated samples, showed high expression of all osteogenic genes but only intermediate expression of YKL-40. Late osteogenesis, the third group, was characterized by peak expression of YKL-40 as well as OCN, RUNX-2 and ALP, but lower expression of OPN.

The increased expression of YKL-40 was correlated to the increased expression of the osteogenic marker genes, with the exception of OPN, which was highest in early glucosamine treated samples (Fig. 2B). Correlation was determined by calculating the Spearman correlation coefficient $\left(\mathrm{r}^{2}\right)$, which was shown to be $77.64 \%$ for YKL-40/RUNX-2, $90.45 \%$ for ALP/RUNX-2 and $78.87 \%$ for OCN/RUNX-2. Visualization by means of a heatmap suggested furthermore that glucosamine might modify osteogenic gene expression to match late osteogenesis compared to control samples. How and why glucosamine is affecting the expression of YKL-40 during long-term cultures was not investigated.

Our data suggest that YKL-40, in combination with increased expression of ALP, RUNX-2 and OCN, could play a role during late osteogenic differentiation of hMSC and this process could be modified by glucosamine, a subunit of the natural substrate for chitinases. YKL-40 is known to take part in tissue remodelling and regeneration but also protects the tissue from negative effects of pro-inflammatory cytokines ${ }^{12}$. This indicates that up-regulation of YKL-40 in developing and regenerating tissues might benefit the remodelling process.

Mineralization. Control cells increased the amount of mineralization deposited over a period of 28 days, as shown in von Kossa staining (Fig. 3A). Glucosamine treatment resulted in an overall impairment of mineral deposition with the first deposits visible after 21 days. Alizarin Red Staining showed the typical increase of calcium deposition expected in control cultures, whereas glucosamine supplemented cells did not show any sign of calcification (Fig. 3B). Photometric evaluation of Alizarin Red Staining visualized the lack of calcification in the presence of glucosamine, while control cells mineralized from day 21 onward (data not shown).

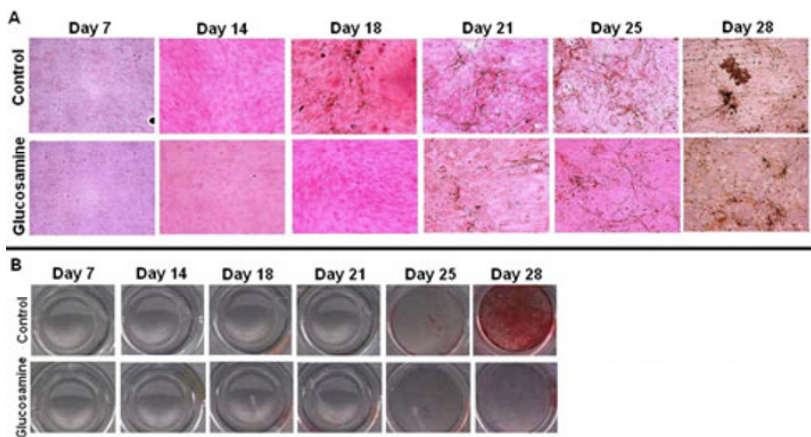

Figure 3. Characterization of mineralization processes during osteogenic differentiation of hMSC. (A) von Kossa Staining of hMSC differentiated for 28 days towards the osteogenic lineage. Dark brown colour is indicative of mineralization. (B) Alizarin Red Staining of hMSC differentiated for 28 days towards the osteogenic lineage. Dark red colour is indicative of calcification. Pictures are representative from 9 experiments.

A likely explanation for the lack of mineralization in glucosamine supplemented cultures could be acidosis of the cell culture media. All glucosamine stock solutions were prepared in PBS, but when the final concentration of glucosamine was added to the cell culture media, a $\mathrm{pH}$ change of the media was observed. Acidosis has been shown to delay mineralization in hMSC-derived osteoblasts and to increase OCN and RUNX-2 expression as well as decrease ALP, osterix (OSX) and OPN gene expression ${ }^{19}$. However, acidosis, as shown by Disthabanchong et al., does not explain the effect of glucosamine on gene expression observed in the present study, apart from the delay in mineralization in $\mathrm{hMSC}^{19}$. ALP mRNA expression and activity have been shown as well by other groups to be increased in the presence of glucosamine, when $\mathrm{pH}$ was stabilized at $7.4^{20}$. Furthermore, when media $\mathrm{pH}$ was adjusted using sodium bicarbonate solution in our study, 
delay in mineralization was resolved (data not shown).

Effect of Glucosamine on Cytokine Expression. Whether the influence of YKL-40 on the activation of the immune system and the tissue remodelling process is due to the proteins themselves or the secondary induction of growth factors and cytokines is not known ${ }^{5}$. Furthermore, increased levels of YKL-40 are believed to protect the tissue from the negative effects of pro-inflammatory cytokines ${ }^{6}$. Therefore, we determined the secretion of several pro- and anti-inflammatory cytokines and growth factors during the osteogenic differentiation of hMSC.

No secretion of IL- $1 \beta$, IL-12p (40), EGF and RANTES was observed in control cells or glucosamine supplemented cells. The anti-inflammatory cytokines IL-4 $(3.9 \pm 2.4 \mathrm{pg} / \mathrm{mL}$ in control vs. $3.5 \pm 2.0 \mathrm{pg} / \mathrm{mL}$ in glucosamine), IL-10 $(8.0 \pm 3.5$ $\mathrm{pg} / \mathrm{mL}$ in control vs. $7.3 \pm 1.1 \mathrm{pg} / \mathrm{mL}$ in glucosamine) and TNF- $\alpha(16.5 \pm 6.9 \mathrm{pg} / \mathrm{mL}$ in control vs. $15.1 \pm 6.4 \mathrm{pg} / \mathrm{mL}$ in glucosamine) were secreted in low amounts over 28 days of osteogenic differentiation (Fig. 4A). The growth factor VEGF $(174.5 \pm 133.1 \mathrm{pg} / \mathrm{mL}$ in control vs. $390.7 \pm 27.7 \mathrm{pg} / \mathrm{mL}$ in glucosamine) showed intermediate secretion and the inflammatory cytokines IL-6 $(1725.6 \pm 307.3 \mathrm{pg} / \mathrm{mL}$ in control vs. $1321.9 \pm 538.2 \mathrm{pg} / \mathrm{mL}$ in glucosamine) and IL-8 (344.6 \pm $133.1 \mathrm{pg} / \mathrm{mL}$ in control vs. $390.7 \pm 165.4 \mathrm{pg} / \mathrm{mL}$ in glucosamine) showed high secretion (Fig. 4B). The differences in secretion levels between control samples and glucosamine treated samples were not statistically significant with any of the cytokines determined.

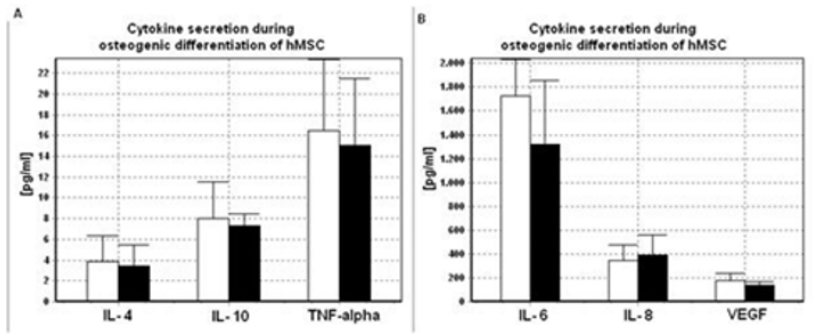

Figure 4. Average cytokine secretion during osteogenic differentiation of hMSC in control media or glucosamine (200 $\mu \mathrm{g} / \mathrm{mL}$ ) supplemented media. (A) Anti-inflammatory cytokines IL-4, IL-10 and TNF- $\alpha$. (B) Inflammatory cytokines IL-6, IL-8 and growth-factor VEGF (average expression over 28 days); error bars describe standard error; $n=2$.

\section{Experimental Section}

Cell Culture. Human, bone-marrow-derived, mesenchymal stromal cells (hMSC) (Lonza, Basel, Switzerland) were cultured in Standard Mesenchymal Stem Cell Basal media (Lonza) supplemented with L-Glutamine, Gentamicin Sulfate/ Amphotericin, and hMSC growth supplement following manufacturer's instructions. For osteogenic differentiation, Differentiation Basal Medium Osteogenic (Lonza) supplemented with dexamethasone, ascorbate, L-Glutamine, Streptamycine/Penicilline, $\beta$-Glycerophosphate and hMSC growth supplement was used. MTT proliferation assays were performed in DMEM/F12 media (Gibco, Carlsbad, California,
USA) supplemented with Penicillin/Streptomycin (Invitrogen) and $10 \%$ hMSC-approved fetal calf serum (Stem Cell, Vancouver, BC, Canada). To determine the effect of glucosamine on hMSC, standard media and osteogenic differentiation media were supplemented with $200 \mu \mathrm{g} / \mathrm{mL}$ Dglucosamine hydrochloride-salt (YSK - Yaizu Suisankagaku Industry, Shizuoka, Japan). Glucosamine stock solutions were prepared by dissolving glucosamine in sterile PBS and filtered through $0.45 \mu \mathrm{m}$ and $0.22 \mu \mathrm{m}$ filters.

To prove multi-lineage differentiation potential, unstimulated cells were differentiated towards the osteogenic, chondrogenic and adipogenic lineage after appropriate expansion. All experiments were done at minimum in triplicate and hMSC from passage 3-5 were used in all experiments.

MTT Proliferation Assays. hMSC were expanded in a 96-well-plate and proliferation determined with the ATCC MTT proliferation kit (ATCC Bioproducts, Boras, Sweden) following manufacturer's instructions.

RNA Isolation and cDNA Transcription. RNA isolation was performed using Qiagen BioRobot workstation (Quiagen, Hilden, Germany) and the EZ-1 RNA Cell Mini Kit (Qiagen), following manufacturer's instructions. High-Capacitiy cDNA Reverse Transcription Kit from Applied Biosystems (Foster City, California, USA) was used in all experiments.

Gene Expression. To quantify gene expression of selected genes, qPCR was performed in a StepOne Real Time PCR System (Applied Biosystems). $10 \mu \mathrm{L}$ aliquots of Taqman master mix and $1 \mu \mathrm{L}$ of Taqman assay (Applied Biosystems) were added to $9 \mu \mathrm{L}$ of 1:10 diluted sample cDNA for each reaction.

Glyceraldehyde 3-phosphate dehydrogenase (GAPDH) was used as a reference gene. Taqman assays included YKL-40 (Hs00609691 m1), acidic mammalian chitinase (AMCase) (Hs00757767_m1), chitotriosidase (CHIT1) (Hs00185753_m1), osteocalcin (OCN) (Hs00609452 g1), osteopontin $(\overline{O P N})$ (Hs00167093 m1), alkaline phosphatase (ALP) (Hs01029141 g1) and runt-related transcription factor 2 (RUNX-2) (Hs00231692_m1), all from Applied Biosystems.

Data were analysed using GenEX 5.3.2.13 software (MultiD Analyses, Gothenburg, Sweden). Data pre-processing was based on averaged qPCR repeats, normalized to the reference gene GAPDH and relative quantities were calculated relative to the earliest control sample (3 days expansion during short-term expansion and 7 days osteogenic differentiation of control samples for osteogenesis experiments). Graphs were created from averaged fold changes over 28 days relative to the respective control sample.

Luminex 10Plex Human Cytokine Assay. Luminex 10Plex Human Cytokine Assay (Panomics, Fremont, California, USA) was performed using media supernatants from stimulated and unstimulated cells during osteogenic differentiation. The following cytokines were analysed: Interleukin- $\beta$ (IL- $1 \beta$, IL-4, IL-6, IL-8, IL-10, IL-12 (p40), tumor necrosis factor- $\alpha$ (TNF- $\alpha$ ), RANTES, vascular endothelial growth factor (VEGF) and epidermal growth factor (EGF) (detection limit of cytokines: $1.3 \mathrm{pg} / \mathrm{mL}$ ).

\section{望 Springer}


Alizarin Red Staining and Quantitation. Cells were washed with phosphate-buffered saline (PBS) and fixed in $4 \%$ paraformaldehyde. Next, cells were washed with distilled $\mathrm{H}_{2} \mathrm{O}$ $\left(\mathrm{dH}_{2} \mathrm{O}\right)$ and stained with a $2 \%$ Alizarin Red Solution (Sigma Aldrich Inc., St. Louis, MO, USA) in $\mathrm{dH}_{2} \mathrm{O}$ at $\mathrm{pH} 4.1$ for 20 min. Cells were then washed with $\mathrm{dH}_{2} \mathrm{O}$ and pictures were taken in a scanner (ScanJet ADF, Hewlett Packard).

For quantification, dried and stained wells were hydrated over-night in $\mathrm{dH}_{2} \mathrm{O}$ and incubated with $10 \%$ cetyl-pyridinium chloride (Sigma) in $\mathrm{dH}_{2} \mathrm{O}$ for $15 \mathrm{~min}$ on a shaker. Cells were spun down and supernatant aliquots were analysed in a MultiSkan spectrometer (Thermo Scientific) at $562 \mathrm{~nm}$.

Von Kossa Staining. Cells were washed with PBS and fixed in $4 \%$ paraformaldehyde. Standard protocols were used to perform von Kossa staining and pictures were taken in a fluorescent microscope (Olympus BX51) using Cell A Imaging Software (Olympus, Center Valley, PA, USA).

Statistical Analysis. Data are presented as means and standard deviations, with the exception of gene expression data and cytokine secretion, where standard error was used. Statistical analysis was performed using GenEx 5.3.2.13 software. To evaluate treatment effect, $t$-test was used and a heatmap based on hierarchical clustering was constructed from autoscaled, pre-processed relative fold changes. Correlation of gene expression data visualized in the heatmap set-up was evaluated by Spearman correlation; $p<0.05$ was considered statistically significant.

\section{Conclusion}

In this study, we showed the expression of the chitinaselike-protein YKL-40 in hMSC, both during expansion of undifferentiated cells and after osteogenic differentiation. Glucosamine did neither affect the maintenance of hMSC pluripotency nor proliferation. The active chitinases, chitotriosidase (CHIT1) and acidic mammalian chitinase (AMCase), were not detected. Glucosamine increased the expression of YKL-40 during osteogenic differentiation, but did not affect expression during short-term expansion. Glucosamine was furthermore associated with an increase in the expression of osteogenic marker genes during osteogenic differentiation of hMSC.

\section{Electronic Supplementary Material}

Supplementary material is available in the online version of this article at http://dx.doi.org/10.1007/s13659-012-0017-0 and is accessible for authorized users.

\section{Acknowledgments}

We would like to thank K Olafsdóttir and S Kristiansdóttir and the staff at the department of pathology, Landspitali-
University Hospital for technical assistance. We would also like to acknowledge SA Hafsteinsson for his contribution and G Stefánsson at the Statistics Center Iceland, University of Iceland, for his assistance with the statistical analysis. This work was supported by grants from Technology Development Fund, managed by the Icelandic Center for Research, and Landspitali University Hospital Research Fund.

Open Access This article is distributed under the terms of the Creative Commons Attribution License which permits any use, distribution, and reproduction in any medium, provided the original author(s) and source are credited.

\section{References}

[1] Sakaguchi, Y.; Sekiya, I.; Yagishita, K.; Muneta, T. Arthritis Rheum. 2005, 52, 2521-2529.

[2] Zuk, P. A.; Zhu, M.; Mizuno, H.; Huang, J.; Futrell, J. W.; Katz, A. J.; Benhaim, P.; Lorenz, H. P.; Hedrick, M. H. Tissue Eng. 2001, 7, 211-228.

[3] Jiang, Y.; Jahagirdar, B. N.; Reinhardt, R. L.; Schwartz, R. E.; Keene, C. D.; Ortiz-Gonzalez, X. R.; Reyes, M.; Lenvik, T.; Lund, T.: Blackstad, M.; Du, J.; Aldrich, S.; Lisberg, A.; Low, W. C.; Largaespada, D. A.; Verfaillie, C. M. Nature 2002, 418, 41-49.

[4] Bleau, G.; Massicotte, F.; Merlen, Y.; Boisvert, C. EXS 1999, 87, 211-221.

[5] Lee, C. G. Yonsei Med. J. 2009, 50, 22-30.

[6] Recklies, A. D.; Ling. H.; White, C.; Bernier, S. M. J. Biol. Chem. 2005, 280, 41213-41221.

[7] Bussink, A. P.; Speijer, D.; Aerts, J. M.; Boot, R. G. Genetics 2007, 177, 959-970.

[8] Renkema, G. H.; Boot, R. G.; Au, F. L.; Donker-Koopman, W. E.; Strijland, A.; Muijsers, A. O.; Hrebicek, M.; Aerts, J. M. Eur. J. Biochem. 1998, 251, 504-509.

[9] Holt, P. G. Toxicol. Lett. 1996, 86, 205-210.

[10] Houston, D. R.; Recklies, A. D.; Krupa, J. C.; van Aalten, D. M. J. Biol. Chem. 2003, 278, 30206-30212.

[11] Hakala, B. E.; White, C.; Recklies, A. D. J. Biol. Chem. 1993 268, 25803-25810.

[12] Ling, H.; Recklies, A. D. Biochem. J. 2004, 380, 651-659.

[13] Sandford, P. A. Advances in Chitin Science; NTNU: Trondheim, 2003; pp. 35-42.

[14] Kirkham, S. G.; Samarasinghe, R. K. J. Orthop. Surg. (Hong Kong) 2009, 17, 72-76.

[15] Meininger, C. J.; Kelly, K. A.; Li, H.; Haynes, T. E.; Wu, G. Biochem. Biophys. Res. Commun. 2000, 279, 234-239.

[16] Ivanovska, N.; Dimitrova, P. Arthritis Res. Ther. 2011, 13, R44.

[17] Dominici, M.; Le Blanc, K.; Mueller, I.; Slaper-Cortenbach, I.; Marini, F.; Krause, D.; Deans, R.; Keating, A.; Prockop, D.; Horwitz, E. Cytotherapy 2006, 8, 315-317.

[18] Bergkvist, A.; Rusnakova, V.; Sindelka, R.; Garda, J. M.; Sjögreen, B.; Lindh, D.; Forootan, A.; Kubista, M. Methods 2010, 50, 323-335.

[19] Disthabanchong, S.; Radinahamed, P.; Stitchantrakul, W.; Hongeng, S.; Rajatanavin, R. Kidney Int. 2007, 71, 201-209.

[20] Ganno, T.; Yamada, S.; Ohara, N.; Matsunaga, T.; Yanagiguchi, K.; Ikeda, T.; Ishizaki, H.; Hayashi, Y. J. Biomed. Mater. Res. A 2007, 82, 188-194. 